\title{
VALORES HUMANOS EXPRESADOS EN ESTUDIANTES DE ENFERMERÍA DE UNA UNIVERSIDAD PÚBLICA DE LA CIUDAD DE ICA, OCTUBRE 2015 - SETIEMBRE 2016
}

\author{
Human values expressed in nursing students of a public university of the city of Ica, \\ October 2015 - September 2016
}

\author{
Isabel Natividad Urure Velazco ${ }^{1 a d}$, Olivia Hermelinda Helia Curasi Gómezª,a, Luisa Antonia Pacheco \\ Villagarcía ${ }^{3 a c}$, Rosario Campos Soto2ac, Fanny Gissela Gabriel Carhuayo4a, \\ Marcia Maritza Gutiérrez Seminario ${ }^{\circ}$
}

\author{
1. Facultad de Enfermería .Universidad Nacional San Luis Gonzaga de Ica, Perú. \\ 2. Hospital Santa María del Socorro. Ica, Perú \\ 3. Hospital Regional de Ica, Perú \\ 4. Hospital Augusto Hernández Mendoza. Ica, Perú \\ a. Licenciada en Enfermería, b. Enfermera especialista en emergencias y desastres, c. Magister en Salud Pública, d. Doctora en Ciencias \\ de la Salud e Estudiantes de Enfermería
}

\section{RESUMEN}

Hoy en día el tema de los valores ocupa un lugar especial en el análisis de la problemática social, y más aún en estudiantes en formación. Objetivo: Determinar los valores humanos expresados por los estudiantes de enfermería en una Universidad Pública de Ica. Material y métodos: Estudio descriptivo, transversal, no experimental constituido por 200 estudiantes del I al VIII Ciclo. Se utilizó una encuesta validado por expertos internacionales y nacionales, que tomaron como base para el instrumento el cuestionario de valores universales de Shalom Schwartz con 40 reactivos tipo escala Likert y 1 reactivo de priorización de 3 valores que se fomentará en la facultad. Resultados: El promedio ponderado global de los puntajes de la escala Likert asignados a los valores expresados por los estudiantes de enfermería fueron de 3,92, observándose los más altos valores en universalidad 4,38, seguridad 4,28, benevolencia 4,22, autodirección 4,19 , y con menos puntajes: Conformidad 3,98, estimulación 3,91, hedonismo 3,83, logro 3,79, tradición 3,72 y poder con 2,89. Se priorizaron 3 valores que se quiere fomentar en la facultad, identificándose como primera prioridad al valor logro con $25,5 \%$, el valor universalidad como segunda prioridad con $17,5 \%$, y seguridad como tercer prioridad con 17,5\%. Conclusiones: La mayoría de estudiantes de la Facultad de Enfermería, expresan que los valores universales de Schwartz son importantes con un $60,5 \%$. Con la contrastación de la hipótesis y aplicando la prueba estadística de Chi Cuadrado $\left(x^{2}\right)$ se acepta la hipótesis de investigación (H1).

Palabras clave: Valores humanos, expresados en estudiantes de enfermería.

\section{SUMMARY}

Today the issue of values has a special place in the analysis of social problems, and even more students in training. Objective: To determine human values expressed by nursing students in a Public University of Ica. Material and Methods: A descriptive, cross-sectional, not experimental study consists of 200 students from I to VIII Cycle. A survey validated by international and national experts, who took as the basis for the instrument questionnaire universal values of Shalom Schwartz with 40 reagents Likert scale, 1 reagent 3 prioritization values promoted in the school was used. Results: The overall weighted average Likert scale scores assigned to the values expressed by nursing students was 3.92 , the highest values were observed in 4.38 universality, safety $4.28,4.22$ benevolence, 4:19 self-direction, and less scores: Compliance 3.98, 3.91 stimulation, hedonism 3,83, 3,79 achievement, tradition and 3.72 to 2.89 . 3 values is to encourage the faculty were prioritized, identified as first priority to achieving value with $25.5 \%$, the universal value as a second priority with 
$17.5 \%$ and third priority security as $17.5 \%$. Conclusions: Most students of the School of Nursing, express the universal values of Schwartz are important with $60.5 \%$. With the testing of the hypothesis and applying the statistical test Chi Square (x2) the research hypothesis $(\mathrm{H} 1)$ is accepted.

Keywords: Human values, expressed in nursing students.

\section{INTRODUCCIÓN}

En la actualidad la importancia del problema de los valores es innegable, la crisis del hombre actual es una crisis de valores que requiere un examen crítico, a fondo, de su naturaleza, sentido, fundamento y jerarquía (1), y que está afectando particularmente a los sectores más jóvenes.

El fenómeno de la globalización ha trastocado la noción clásica del espacio - tiempo en el que se apegaban tradicionalmente las relaciones humanas (2). Los estudiantes de enfermería no viven ajenos a este fenómeno, observado a nivel mundial de crisis de valores.

En este contexto," en el caso de las instituciones de educación superior de enfermería, el interés por la formación en valores parte de que tienen como misión formar profesionales de enfermería humanistas, con capacidad innovadora, regidos por el pensamiento crítico, que sean líderes en el cuidado profesional" (3). La formación de valores es tan importante como el propio contenido que se imparte en cada una de las asignaturas, se trata por lo tanto, que el sistema de conocimientos y habilidades tengan implícito los valores que se requieren formar (4). De esta manera," el conocimiento y la formación ética de profesional de enfermería debe fortalecerse durante sus etapas de formación, tanto en pregrado como en postgrado, ya que el enfermero trabaja bajo la influencia de una escala de valores profesional y personal cuando entra en relación con los pacientes, y en algunos momentos los valores del profesional de enfermería pueden entrar en conflicto con los del paciente" (5). Entendiéndose como valor como "algo que vale la pena, una creencia estimada por una persona, o una disposición afectiva hacia una persona, objeto o idea". Los valores dan dirección y significado a la vida y guían el comportamiento de una persona (6). Una persona que ingresa a la enfermería con un juego de valores personales, adquiere valores profesionales que influencian las acciones de enfermería a través de la socialización en la escuela y en los lugares de práctica. Es importante para las enfermeras poner en claro los valores personales para entender como éstos guían las acciones e influencian la toma de decisiones (6). Por consiguiente se puntualiza que "la ética personal surge de los valores personales" (7). Es así que "día a día las enfermeras se vuelven más conscientes de los valores y derechos de los pacientes, así como de las personas que los apoyan y de la ética que involucra la práctica de enfermería (6). En este sentido, haciendo una revisión bibliográfica, se señala que investigadores preocupados por el problema de los valores, han realizado estudios con el fin de medir estos aspectos, uno de ellos es la Escala de Valores Universales de Shalom Schwartz etc. Asimismo se reporta estudios como en Cuba donde se aprecia "de manera general hubo $56,5 \%$ de respuestas correctas sobre los conceptos importantes de la formación de valores" (8) deduciendo una brecha importante de $43,5 \%$ en relación a este tema.

Por todos estos considerandos analizados y observados a nivel mundial, consideramos que la temática del problema de valores es un fenómeno que también se evidencia en nuestro medio y la preocupación es que los estudiantes de enfermería en su accionar de este futuro profesional es el de proporcionar el cuidado dirigido a seres humanos. De ahí la necesidad de investigar los valores existentes en los estudiantes de enfermería de la Universidad Pública de Ica, y determinar la 
presencia o ausencia de valores, así como establecer estrategias encaminadas a promover y fomentar la formación de valores conducentes al establecimiento de una base sólida en la escala de valores.

Teniendo en cuenta la importancia del tema porque en el proceso educativo universitario el rol de generación y difusión del conocimiento esto no es todo. La universidad en su actividad formadora y de investigación incorpora contenidos éticos propios para cada profesión de forma que el futuro profesional, además de lograr ser experto en su materia, se encuentre en condiciones de actuar con base a criterios éticos. Siendo también de trascendencia este estudio porque dada la prioridad del tema, en la actualidad, hay poca información de tesis u artículos científicos, relacionadas con el estudio, el cual servirá de remanente teórico para futuros trabajos de investigación, el mismo que va a conducir a identificar debilidades y establecer estrategias que fortalezcan la escala de valores de los estudiantes en formación, señalándose como objetivo determinar los valores humanos expresados por los estudiantes de enfermería en una Universidad Pública de Ica.

\section{MATERIAL Y MÉTODOS.}

El estudio hizo uso del método descriptivo, diseño no experimental de corte transversal, efectuándose en la Facultad de Enfermería de la Universidad Nacional "San Luis Gonzaga" de Ica, ubicado en la Panamericana Sur Km 305 Av. Los Maestros S/N Ica, Perú - Ciudad Universitaria. Con una población de 780 estudiantes y muestra constituida por 200 estudiantes del I al VIII Ciclo de estudios. Se determinó el tamaño muestral por conveniencia. Se excluyeron los ciclos superiores IX y X Ciclo porque se encontraban realizando prácticas pre-profesionales (Internado), utilizando un cuestionario que estuvo estructurado en 3 partes con 41 reactivos:

- Primera parte: Datos personales 0 sociodemográficos de los estudiantes de la
Facultad de Enfermería, referente a: Edad, sexo, ciclo de estudios, tipo de colegio de procedencia, religión, convivencia familiar, número de hermanos y condición laboral.

- Segunda parte: Corresponde al cuestionario de valores de Schwartz (PVQ) es el instrumento que se desarrolló con la finalidad de evaluar los valores. Este instrumento cuenta con 40 reactivos cortos elaborados por García Del Junco et al (9) adaptado a nuestro medio, dentro de los cuales están los 10 valores universales de Shalom Schwartz, el Portrait Values Questionnaire (PVQ) estudia los siguientes valores, distribuidos en los ítems correspondientes

1. Benevolencia: 4 reactivos (ítems $12,18,27,33$ )

2. Universalidad: 6 reactivos (ítems $3,8,19,23,29$, 40)

3. Autodirección o: 4 reactivos (ítems $1,11,22,34$ )

4. Estimulación : 3 reactivos ( ítems $6,15,30$ )

5. Hedonismo: 3 reactivos (ítems $10,26,37$ )

6. Logro: 4 reactivos (ítems $4,13,24,32$ )

7. Poder: 3 reactivos (ítems $2,17,39$ )

8. Seguridad: 5 reactivos (ítems $5,14,21,31,35$ )

9. Conformidad: 4 reactivos (ítems $7,16,28,36$ )

10. Tradición: 4 reactivos (ítems $9,20,25,38$ )

- Tercera Parte: Priorización de 3 valores fundamentales de Schwartz que se fomentarán en los estudiantes de enfermería, tomándose como base el constructo de Lucía García Naranjo (10), modificado y adaptado a nuestra realidad.

Medida la segunda parte (40 reactivos cerradas de opción múltiple (tipo escala Likert) utilizando una escala de alternativas cuyas puntuaciones son:

5. Totalmente de acuerdo

4. De acuerdo

3. Ni de acuerdo ni en desacuerdo

2. En desacuerdo

1. Totalmente en desacuerdo

En la recolección de datos se empleó como técnica la encuesta y como instrumento el cuestionario de García Del Junco et al, realizado en Perú (9) que fue validado por expertos internacionales y aplicada a trabajos de investigación sobre valores en Lima, Perú , 
teniendo como base los 10 valores universales de Schwartz. Se reestructuró los reactivos adaptándolo a nuestra realidad, previa prueba piloto. Se hizo uso del programa de análisis estadístico de datos SPSS versión 21, para la prueba de hipótesis se aplicaron las pruebas de Chi cuadrado $\left(\mathrm{X}^{2}\right)$ para la relación entre variables nominales dicotómicas o politómicas, y la prueba de U-Mann-Withney para la contrastación entre variables dicotómicas y ordinales. Para la contrastación de la hipótesis se utilizó la prueba estadística de Chi cuadrado $\left(X^{2}\right)$.

\section{RESULTADOS.}

Durante el periodo de investigación, se logró entrevistar 200 alumnas y alumnos de la Facultad de Enfermería de la Universidad Nacional "San Luis Gonzaga" de Ica, a quienes se aplicó el cuestionario de valores de Schwartz (PVQ) durante el trabajo de campo realizado entre Marzo a Mayo 2016 ; la muestra se seleccionó equitativamente entre el Primero y Octavo Ciclos de Estudios (25, $12.5 \%$, por cada uno) y estuvo constituida principalmente por estudiantes de sexo femenino (92,0\%), de 15 a 24 años de edad $(96,5 \%)$, procedentes de colegios públicos $(68,5 \%)$, de religión católica $(84,5 \%)$, que vivían con ambos padres (68,0\%), que tenían de 1 a 3 hermanos $(75,0 \%)$ y dedicados exclusivamente a estudiar $(86,5 \%)$.

Se pesquisó la expresión de los 10 valores universales de Schwartz por parte de los alumnos encuestados, observándose que el promedio ponderado más alto según la escala Likert resultó en prioridad al valor universalidad con 4,38 seguido luego en orden decreciente los valores: Seguridad con 4,28 , Benevolencia con 4,22, autodirección o autodeterminación 4,19 , conformidad 3,98 , estimulación 3,91 , hedonismo 3,83 , logro 3,79, tradición 3,72 y por último el vapor poder con 2,89 de promedios ponderados según escala Likert en sus ítems respectivos. Siendo el promedio ponderado global de los puntajes de las escalas Likert asignados a los valores expresados por los estudiantes de la Facultad de Enfermería de la Universidad Nacional San Luis Gonzaga de Ica, en el estudio fue de 3,92 (Tabla $N^{\circ} 1$ ). Los estudiantes reconocieron como importantes los valores estudiados el $60,5 \%$ y como no importantes $39,5 \%$ (Tabla N²)

Los tres valores priorizados por los estudiantes de enfermería que se quiere fomentar en la facultad, señalaron al valor: Logro $(25,5 \%)$, universalidad (18,5\%) y seguridad $(17,5)$, como primera, segunda y tercera prioridad respectivamente (Tabla $\mathrm{N}^{\circ} 3$ ).

Relación entre las características sociodemográficas y la escala de valores expresados por los estudiantes de enfermería se pudo determinar una asociación entre la expresión de valores importantes con las categorías sexo femenino $(56,0 \%)$, grupo etario 20 a 24 años $34,0 \%$, cursar el sexo ciclo $10,0 \%$, proceder de colegio público $42,0 \%$, profesar religión católica $50,5 \%$, vivir con ambos padres $40,0 \%$, tener 1 a 3 hermanos $45 \%$ y no trabajar $52,5 \%$ de estas asociaciones la única que resultó estadísticamente significativa fue la de cursar el sexto ciclo de estudios $(P \leq 0,05)$ .Aplicándose como pruebas hipótesis Chi Cuadrado para la relación entre variables nominales dicotómicas o politómicas y la prueba de U- Mann whitney para la contrastación entre variables politómicas ordinales (Tabla $\mathrm{N}^{\circ} 4$ ).

Tabla $N^{\circ}$ 1: Promedios ponderados de los puntajes de la Escala de Likert según valores expresados por los encuestados 


\begin{tabular}{lc}
\hline \multicolumn{1}{c}{ Valores } & $\begin{array}{c}\text { Promedio } \\
\text { ponderado }\end{array}$ \\
\hline Universalidad & 4,38 \\
Seguridad & 4,28 \\
Benevolencia & 4,22 \\
Autodirección & 4,19 \\
Conformidad & 3,98 \\
Estimulación & 3,91 \\
Hedonismo & 3,83 \\
Logro & 3,79 \\
Tradición & 3,72 \\
Poder & 2,89 \\
\hline \multicolumn{1}{c}{ Promedio $\quad$ global } & 3,92 \\
\hline
\end{tabular}

Tabla N²: Distribución porcentual de los encuestados según categorías de valores

\begin{tabular}{ccc}
\hline Respuesta & $\mathrm{n}$ & $\%$ \\
\hline Importantes & 121 & 60,5 \\
No importantes & 79 & 39,5 \\
\hline Total & 200 & 100 \\
\hline
\end{tabular}

Fuente: Base de datos.

Prueba de hipótesis: X²: 8,82; gl: 1; p: 0,003.

Tabla $\mathbf{N}^{\circ}$ 3: Distribución porcentual de los encuestados según escala de priorización de los valores.

\begin{tabular}{|c|c|c|c|c|c|c|c|c|}
\hline \multicolumn{3}{|c|}{ Primera prioridad } & \multicolumn{3}{|c|}{ Segunda prioridad } & \multicolumn{3}{|c|}{ Tercera prioridad } \\
\hline Valor & $\mathrm{n}$ & $\%$ & Valor & $\mathrm{n}$ & $\%$ & Valor & $\mathrm{n}$ & $\%$ \\
\hline Logro. & 51 & 25,5 & Universalidad & 37 & 18,5 & Seguridad. & 35 & 17,5 \\
\hline Universalidad. & 46 & 23,0 & Logros. & 35 & 17,5 & Tradición & 27 & 13,5 \\
\hline Benevolencia & 32 & 16,0 & Seguridad. & 32 & 16,0 & Autodeterminación & 25 & 12,5 \\
\hline Autodeterminación & 31 & 15,5 & Benevolencia & 31 & 15,5 & Logros. & 24 & 12,0 \\
\hline Seguridad. & 17 & 8,5 & Autodeterminación & 18 & 9,0 & Benevolencia & 23 & 11,5 \\
\hline Tradición & 7 & 3,5 & Tradición & 15 & 7,5 & Poder social. & 19 & 9,5 \\
\hline Poder social. & 6 & 3,0 & Poder social. & 10 & 5,0 & Universalidad & 18 & 9,0 \\
\hline Hedonismo. & 5 & 2,5 & Conformidad. & 10 & 5,0 & Estimulación & 14 & 7,0 \\
\hline Conformidad. & 5 & 2,5 & Estimulación & 9 & 4,5 & Conformidad. & 9 & 4,5 \\
\hline Estimulación & 0 & 0,0 & Hedonismo. & 3 & 1,5 & Hedonismo. & 6 & 3,0 \\
\hline Total & 200 & 100,0 & Total & 200 & 100.0 & Total & 200 & 100,0 \\
\hline
\end{tabular}

Fuente: Base de datos.

Tabla $\mathbf{N}^{\circ}$ 4: Distribución de los encuestados según categorías de valores y variables sociodemográficas 


\begin{tabular}{|c|c|c|c|c|c|c|}
\hline \multirow{2}{*}{ Valores } & \multirow{2}{*}{\multicolumn{4}{|c|}{ Categoría de valores. }} & \multirow{2}{*}{\multicolumn{2}{|c|}{ Prueba de hipótesis }} \\
\hline & & & & & & \\
\hline & \multicolumn{4}{|c|}{$N=200$} & & \\
\hline & \multicolumn{2}{|c|}{ Importantes } & \multicolumn{2}{|c|}{ No importantes } & \multirow[b]{2}{*}{ Valor $p$} & \multirow[b]{2}{*}{ Interpretación } \\
\hline Sexo & $\mathrm{n}$ & $\%$ & $\mathrm{n}$ & $\%$ & & \\
\hline Femenino & 112 & 56,0 & 72 & 36,0 & $\left.0,071^{*}\right)$ & NS \\
\hline Masculino & 9 & 4,5 & 7 & 3,5 & & \\
\hline Grupos etarios & $\mathrm{n}$ & $\%$ & $\mathrm{n}$ & $\%$ & Valor $p$ & Interpretación \\
\hline 15 a 19 & 48 & 24,0 & 47 & 23,5 & $0,288^{(* *)}$ & NS \\
\hline 20 a 24 & 68 & 34,0 & 30 & 15,0 & & \\
\hline 25 a 29 & 5 & 2,5 & 2 & 1,0 & & \\
\hline Ciclo de estudios & $\mathrm{n}$ & $\%$ & $\mathrm{n}$ & $\%$ & Valor $p$ & Interpretación \\
\hline Primero & 7 & 3,5 & 18 & 9,0 & $0,002^{(* *)}$ & $S$ \\
\hline Sequndo & 15 & 7,5 & 10 & 5,0 & & \\
\hline Tercero & 12 & 6,0 & 13 & 6,5 & & \\
\hline Cuarto & 17 & 8,5 & 8 & 4,0 & & \\
\hline Quinto & 14 & 7,0 & 11 & 5,5 & & \\
\hline Sexto & 20 & 10,0 & 5 & 2,5 & & \\
\hline Séptimo & 18 & 9,0 & 7 & 3,5 & & \\
\hline Octavo & 18 & 9,0 & 7 & 3,5 & & \\
\hline $\begin{array}{c}\text { Colegio de } \\
\text { procedencia }\end{array}$ & $\mathrm{n}$ & $\%$ & $\mathrm{n}$ & $\%$ & Valor $\mathrm{p}$ & Interpretación \\
\hline Privado católico & 31 & 15,5 & 21 & 10,5 & $0,897^{(*)}$ & NS \\
\hline Privado laico & 6 & 3,0 & 5 & 2,5 & & \\
\hline Público & 84 & 42,0 & 53 & 26,5 & & \\
\hline Religión & $\mathrm{n}$ & $\%$ & $\mathrm{n}$ & $\%$ & Valor $\mathrm{p}$ & Interpretación \\
\hline Católico & 101 & 50,5 & 68 & 34,0 & $0,765^{(*)}$ & NS \\
\hline Evangélico & 7 & 3,5 & 5 & 2,5 & & \\
\hline Otro & 9 & 4,5 & 3 & 1,5 & & \\
\hline Ninguna & 4 & 2,0 & 3 & 1,5 & & \\
\hline Convivencia familiar. & $\mathrm{n}$ & $\%$ & $\mathrm{n}$ & $\%$ & Valor $\mathrm{p}$ & Interpretación \\
\hline Ambos padres & 80 & 40,0 & 56 & 28,0 & $0,134^{(*)}$ & NS \\
\hline Solo padre & 6 & 3,0 & 7 & 3,5 & & \\
\hline Solo madre & 22 & 11,0 & 14 & 7,0 & & \\
\hline Solo & 13 & 6,5 & 2 & 1,0 & & \\
\hline $\begin{array}{l}\text { Número de } \\
\text { hermanos. }\end{array}$ & $\mathrm{n}$ & $\%$ & $\mathrm{n}$ & $\%$ & Valor $p$ & Interpretación \\
\hline Ninguno & 9 & 4,5 & 10 & 5,0 & $0,099^{(* *)}$ & NS \\
\hline Uno & 31 & 15,5 & 35 & 17,5 & & \\
\hline Dos & 30 & 15,0 & 14 & 7,0 & & \\
\hline Tres & 29 & 14,5 & 11 & 5,5 & & \\
\hline Más de tres & 22 & 11,0 & 9 & 4,5 & & \\
\hline $\begin{array}{l}\text { Condición laboral o } \\
\text { trabajo actual. }\end{array}$ & $\mathrm{n}$ & $\%$ & $\mathrm{n}$ & $\%$ & Valor $p$ & Interpretación \\
\hline Sí trabajo & 16 & 8,0 & 11 & 5,5 & $\left.0,887^{*}\right)$ & NS \\
\hline No trabajo & 105 & 52,5 & 68 & 34,0 & & \\
\hline
\end{tabular}

Fuente: Base de datos.

Pruebas de hipótesis: $\left.{ }^{*}\right) X^{2} ;\left(^{* *}\right) \cup$ de Mann Whitney

Siglas: $\mathrm{S}=$ Significativo; NS= No Significativo. 


\section{DISCUSIÓN.}

Hoy el día el tema de los valores ocupa un lugar especial en el ámbito de la problemática social, evidenciamos una crisis de valores en este mundo globalizado que han trastocado las relaciones humanas, siendo esta situación preocupante, no ajenos a esta realidad está el profesional de enfermería y estudiantes en formación, que por su propia naturaleza tienen que brindar cuidados integrales y/o holísticos a seres humanos, considerando que el enfermero trabaja bajo la influencia de una escala de valores. En este contexto, haciendo una revisión bibliográfica se señala que investigadores preocupados por el problema de los valores, han realizado estudios con el fin de medir estos aspectos; uno de ellos es la Escala de Valores Universales de Shalom Schwartz, etc. Asimismo en Cuba reporta Rodríguez et al. en su estudio que "de manera general hubo $56,5 \%$ de respuestas correctas sobre conceptos importantes de la formación de valores" (8), en enfermeros. Este resultado es coincidente con el estudio efectuado, que mostró un $60,5 \%$ que reconocieron los valores como importantes, existiendo todavía una brecha importante de 39,5\% por alcanzar el nivel óptimo deseado en la escala de valores en estudiantes de enfermería en la muestra que fue sujeto a estudio.

En relación a los hallazgos referentes a la priorización de la escala de valores humanos expresados por los estudiantes de enfermería, en el primer grupo nos muestra que ubicaron en los 3 primeros lugares a universalidad, seguridad y benevolencia, dándole un promedio ponderado casi similar de 4,38, 4,28 y 4,22 respectivamente. Coincidente este resultado con García J. (9), en su estudio respecto a la escala de valores deseados que ubicaron de la siguiente manera: Seguridad, universalidad y benevolencia, observándose ligera diferencia de ubicación los 2 primeros, pero ambos consideraron a benevolencia en 3er lugar. Asimismo se puede señalar que es también coincidente las cifras con el modelo de
Schwartz (estructura circular de los valores 1992) en la que universalidad y benevolencia son valores de autotrascendencia y seguridad, conformidad, etc., son valores conservativos. De la misma forma se puede puntualizar que García L. (10), en su estudio investigativo ubica en su perfil valorativo en los 3 primeros lugares a autodeterminación o autodirección, benevolencia y universalismo, similar a la muestra en estudio que ubica a benevolencia y universalismo dentro de los 3 primeros valores priorizados. No siendo coincidente estos resultados con Grimaldo (11), que ubican al poder y tradición como el primero y segundo valor más importante. En este sentido se puede apreciar que los resultados del estudio reflejan que los estudiantes destacan la aceptación de otros como iguales, así como la preocupación por su bienestar dentro de los cuales está los valores priorizados (universalidad y benevolencia) mostrando la preocupación de trascender de sus intereses particulares en beneficio de los intereses de la sociedad, así como conservar la armonía y estabilidad de la sociedad dentro del cual está el valor priorizado (seguridad).

Luego los valores priorizados como segundo grupo fueron: Autodeterminación o autodirección, conformidad, estimulación y hedonismo como promedios ponderados de $4,19,3,98,3,91$ y 3,83 respectivamente. Siendo los resultados similares con García J. (9), que ubicaron a los valores en el siguiente orden en la escala de valores deseados: Tradición, estimulación, hedonismo y conformidad, pudiendo apreciar que solo hay diferencia 0 no es coincidente en el primer lugar. Estas cifras muestran valores que propician la apertura o la independencia, juicio y acción que favorecen al cambio dentro de los cuales se señala los valores priorizados (autodirección, estimulación y hedonismo) y se inclinan al respecto a normas (conformidad), considerando que la labor del profesional de enfermería tiene como base sólida regida al respeto de normas y valores ético morales, las que se debe promover y fomentar. 
El tercer grupo o componente de valores priorizados por los estudiantes señalaron: logro, tradición y poder dándole un promedio ponderado de 3,79 ,3,72 y 2,89 consecutivamente, estos resultados fueron casi similares con el estudio de García J. ( 9 ) con respecto a logro y poder que ubicaron como tercer componente incluyendo también a autoderminación en este grupo, no siendo coincidentes este valor y tradición que lo ubico el autor en mención en el segundo componente Asimismo es coincidente con Schwartz 1992 (12), respecto a ubicar al valor de logro y poder como valores de autopromoción que enfatizan la búsqueda del éxito personal, se puede apreciar estas cifras refuerzan los resultados, en que los estudiantes posponen sus intereses personales por la satisfacción y bienestar de los demás, tal como lo puntualiza la estructura circular de los valores correspondientes a auto promoción ,lo que demuestra que la mayoría de los estudiantes se guían y tienen como base una escala de valores, lo que es beneficioso para el futuro profesional.

Con lo que podemos concluir que los estudiantes reconocieron la escala de los 10 valores estudiados como importantes $60,05 \%$, existiendo todavía una brecha de $39,95 \%$, con un promedio ponderado general de 3,92 , con un déficit de promedio de 1,08, para alcanzar lo óptimo requerido del $100 \%$ y de un puntaje o rango de 5 respectivamente.

\section{Correspondencia:}

Dra .Isabel Natividad Urure Velazco

Correo electrónico: isabelnauv@yahoo.com

\section{REFERENCIAS BIBLIOGRÁFICAS}

1. Gonzáles A. El enfoque centrado en la persona. México: Ed. Trillas; 1991.
2. De Farramiñam J. El desafío de los valores éticos en un mundo globalizado. Universidad de Jaén. Observatorio de la Globalización. Jaén, Perú; 2009.

3. Díaz M, Castro D, Cuevas J. Valores profesionales de enfermería: Una mirada hacia la formación en la Educación Superior. Humanidades Médicas. 2012; 12(2): 292.

4. Rodríguez S, Zulueta F. La formación de valores en el estudiante universitario. Una reflexión y un reto. Gaceta Médica Espirituana 2001; 3(1) Cuba.

5. Hernández A. Algunas consideraciones acerca de los valores humanos y el profesional de enfermería. Aquichan; 1(1): 18. Colombia; 2001.

6. Kozier B, Erb Gle, Bufalino P. Fundamentos de Enfermería. México: Ed. Interamericana McGraw-Hill; 2001. Cap. 5. P 69-82.

7. Potter P, Perry A. Fundamentos de Enfermería. $6^{\circ}$ ed. Volumen I. España: Ed. Grafos; 2007: Capítulo 20, p. 431, 418.

8. Rodriguez C, Piloto K, Insúa L, Morejón B. Formación de valores en los enfermeros del nuevo modelo pedagógico. Pinar del Rio, Cuba. Rev. Ciencias Médicas 2007; 11 (4).

9. García J, Medina E, Dutschke G. Una revisión exploratoria del Modelo de Schwartz. (An exploratory revition of Schwartz's model) Cali, Colombia. Rev. Economía, Gestión y Desarrollo. 2010. (9). p 34-68

10. García L. Sistema Valorativo de Estudiantes de Pregrado y Preuniversitarios de la Pontificia Universidad Católica del Perú. Tesis Pregrado. Pontificia Universidad Católica del Perú. Facultad de Letras y Ciencias Humanas. Departamento de Psicología. Lima, Perú; 2005.

11. Grimaldo $\mathbf{M}$, Merino $\mathbf{C}$. Valores en un grupo de estudiantes de psicología de una Universidad particular de la ciudad de Lima, Perú Rev. Liber. 2009; $15(.1)$ 
Cultura y Educación. Edit. Pearson

Prentice Hall. Madrid, España; 2004.

12. Zlobina, A. La Teoría de Valores de $S$. Schwartz. En D. Páez; I, Fernández; S.

Ubillos y E. Zubieta. Psicología Social,

Recibido: 30/09/16

Aprobado para Publicación: 01/12/2016 